\title{
Investigational drugs in early phase clinical trials targeting thermotransient receptor potential (thermoTRP) channels
}

Asia Fernández-Carvajal , Rosario González-Muñiz , Gregorio FernándezBallester \& Antonio Ferrer-Montiel

To cite this article: Asia Fernández-Carvajal , Rosario González-Muñiz , Gregorio FernándezBallester \& Antonio Ferrer-Montiel (2020): Investigational drugs in early phase clinical trials targeting thermotransient receptor potential (thermoTRP) channels, Expert Opinion on Investigational Drugs, DOI: 10.1080/13543784.2020.1825680

To link to this article: https://doi.org/10.1080/13543784.2020.1825680

\section{曲 Published online: 29 Sep 2020.}

Submit your article to this journal 줄

ШII Article views: 31

Q View related articles $\longleftarrow$

View Crossmark data $₫$ 


\title{
Investigational drugs in early phase clinical trials targeting thermotransient receptor potential (thermoTRP) channels
}

\author{
Asia Fernández-Carvajala ${ }^{a}$ Rosario González-Muñiz ${ }^{\mathrm{b}}$, Gregorio Fernández-Ballester ${ }^{\mathrm{a}}$ and Antonio Ferrer-Montiel ${ }^{\mathrm{a}}$
}

aInstituto De Investigación, Desarrollo E Innovación En Biotecnología Sanitaria De Elche (Idibe), Universitas Miguel Hernández, Alicante, Spain;

bInstituto De Química Médica, CSIC, Madrid, Spain

\begin{abstract}
Introduction: Thermo transient receptor potential (thermoTRP) channels are some of the most intensely pursued therapeutic targets of the past decade. They are considered promising targets of numerous diseases including chronic pain and cancer. Modulators of these proteins, in particular TRPV1-4, TRPM 8 and TRPA1, have reached clinical development, but none has been approved for clinical practice yet.

Areas covered: The therapeutic potential of targeting thermoTRP channels is discussed. The discussion is centered on our experience and on available data found in SciFinder, PubMed, and ClinicalTrials.gov database from the past decade. This review focuses on the therapeutic progress concerning this family of channels, including strategies to improve their therapeutic index for overcoming adverse effects.

Expert opinion: Although thermoTRPs are pivotal drug targets, translation to the clinic has faced two key problems, (i) unforeseen side effects in Phase I trials and, (ii) poor clinical efficacy in Phase II trials. Thus, there is a need for (i) an enhanced understanding of the physiological role of these channels in tissues and organs and (ii) the development of human-based pre-clinical models with higher clinical translation. Furthermore, progress in nanotechnology-based delivery strategies will positively impact thermoTRP human pharmacology.
\end{abstract}

ARTICLE HISTORY

Received 15 June 2020

Accepted 15 September 2020

\section{KEYWORDS}

Cancer; clinical trials; inflammation; nociception; pain; thermoTRPs channels; TRPV1-4; TRPM8; TRPA1

\section{Introduction}

TRP channels function as communication hubs in cells transducing chemical and physical stimuli into a diversity of cellular responses. These ionotropic receptors respond to multiple environmental stimuli allowing the flow of cations down their electrochemical gradient. In neurons, activation of TRP channels depolarizes the plasma membrane promoting action potential generation. In non-excitable cells, these channels are involved in a myriad of processes including cell proliferation, differentiation, and signaling.

TRP channels are expressed in a wide range of species from yeast to humans and currently represent a large family of 28 members grouped in 8 subfamilies, namely canonical (TRPC), vanilloid (TRPV), ankyrin (TRPA), melastatin (TRPM), mucolipin (TRPML), mechano (TRPN), polycystin (TRPP), and one family identified in yeast (TRPY) [1]. Despite the large diversity of biophysical and pharmacological properties of TRP family members, a common physiological ground is their implication in sensory functions from thermo- and mechanosensation to vision and taste. Their implication in detecting potentially noxious environmental signals makes them pivotal cellular sensors for animal behavior and survival in a changing environment.

The range of TRP channel functions is corresponded by an overall conserved atomic structure that is tuned by the diverse architecture of their intracellular modular domains. TRP channels share a tetrameric assembly, which is wellpreserved in detergent, amphipol, or reconstituted into nanodiscs [2]. Subunits usually assemble into homotetramers but can also form heterotetramers, which can have important implications to develop specific pharmacological modulators [3]. There is relatively low sequence homology between family members and the overall structure of the channels can diverge significantly. Even though this divergence in their overall structure, all members share a subunit domain organization consisting of six-transmembrane helices (S1 through S6) with a reentrant loop between S5 and S6 that forms the channel pore including the selectivity filter [4]. The cytosolic $\mathrm{N}$-terminus may contain ankyrin repeats or TRPM homology regions, and phosphorylation consensus sites for serine/threonine and tyrosine kinases. The $\mathrm{C}$-terminus may contain a calmodulin ( $\mathrm{CaM}$ ) and tubulin binding regions, consensus sequences for protein kinases, and a highly conserved 25-mer region, known as the TRP domain. This region has been implicated in channel oligomerization and appears to be critical for functionally coupling stimuli sensing to pore opening [5].

To date, up to 11 TRP channels have been identified in mammals with thermosensitive abilities (referred to as thermoTRPs) (Figure 1). ThermoTRPs belong to the TRPV, TRPM, TRPA, and TRPC subfamilies, and their temperature thresholds for activation are in the range of physiological temperatures that humans can discriminate. TRPV1, TRPV2, 


\section{Article highlights}

- ThermotRPs ion channels are validated therapeutic targets for numerous diseases including chronic pain to cancer.

- Modulators of these proteins, in particular TRPV1-4, TRPM8 and TRPA1, have reached clinical development, but their safety and efficacy are still a main issue.

- Poor clinical translation of preclinical results has precluded the progress of thermoTRP channel modulators as therapeutics.

- The development of human-based preclinical models for thermotRP dysfunction will enhance the clinical translation of drug candidates.

- Topical application of thermoTRP modulators for skin disorders appears as a therapeutic strategy devoid of unwanted side effects associated with systemic distribution.

This box summarizes the key points contained in the article. gastrointestinal, skin, sensory, bladder, cardiac, and neural systems (Figure 2). The involvement of thermoTRP channels in so many pathological processes has made them pharmacological targets of great interest for the pharmaceutical industry. This is supported by (i) therapeutic validation in animal models of human diseases and, (ii) the progress of small molecules targeting thermoTRPs to clinical trials for a variety of diseases [6].

Here we review the current progress of investigational drugs in early phase clinical trials targeting thermoTRP channels. Among the 11 members of this family, only compounds against six of them have reached clinical trials, the majority targeting TRPV1 (11 drugs) followed by TRPM8 (five), TRPA1 (four), TRPV2 (two), TRPV3 (one), and TRPV4 (one). TRPM3 is also actively pursued but no drug candidates have been reported yet to reach clinical trials.

\section{ThermoTRP channels}

\subsection{TRPV subfamily}

The TRPV subfamily comprises six members that can be divided into two groups based in their sequence homology: TRPV1-4 and TRPV5-6 (Figure 1). The name vanilloid comes from capsaicin, the pungent vanilloid compound present in chili peppers, that binds and gates TRPV1 channels (previously known as the capsaicin receptor). All four channels within the TRPV1-4 group are polymodal channels also gated by warm a dysfunction of some of these channels in respiratory,

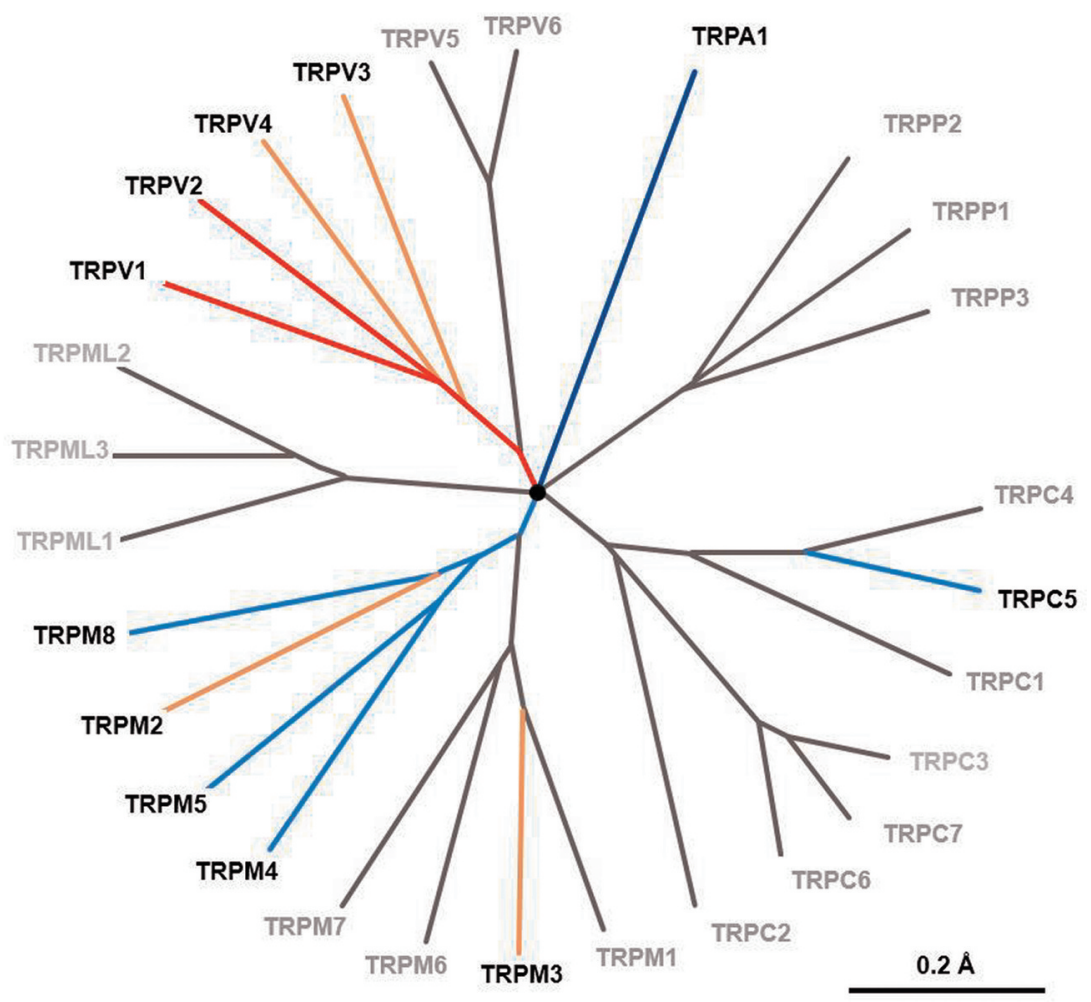

Figure 1. Phylogenic tree of human TRP channels. On the basis of sequence homology, all TRP channels fall into seven subfamilies that comprise proteins with distinct channel properties. The thermo-TRP are represented by colors based on their temperature activation range, in deep red appear those channels activated by noxious heat (TRPV $2 \geq 52^{\circ} \mathrm{C}$; TRPV $1 \geq 43^{\circ} \mathrm{C}$ ), in orange those activated by warmth temperatures $\left(\mathrm{TRPM} 2 \geq 37^{\circ} \mathrm{C} ; \mathrm{TRPV}^{\circ} \geq 36^{\circ} \mathrm{C} ; 30^{\circ} \mathrm{C} \leq \mathrm{TRPM} 3 \geq 35^{\circ} \mathrm{C}\right.$ and $\mathrm{TRPV} 4 \leq 34^{\circ} \mathrm{C}$ ) in light blue channels activated by cold $\left(25^{\circ} \mathrm{C} \leq \mathrm{TRPC} 5 \leq 37^{\circ} \mathrm{C}\right.$; TRPM $\left.8 \leq 28^{\circ} \mathrm{C}\right)$, and the one activated by noxious cold (TRPA $\left.1 \leq 17^{\circ} \mathrm{C}\right)$ is represented by deep blue. The bar (0.2) indicates point accepted mutation units, which is the evolutionary distance between two amino acids ( 1 point accepted mutation unit = 1 point mutation event per 100 amino acids, which is accepted and is passed to progeny). 


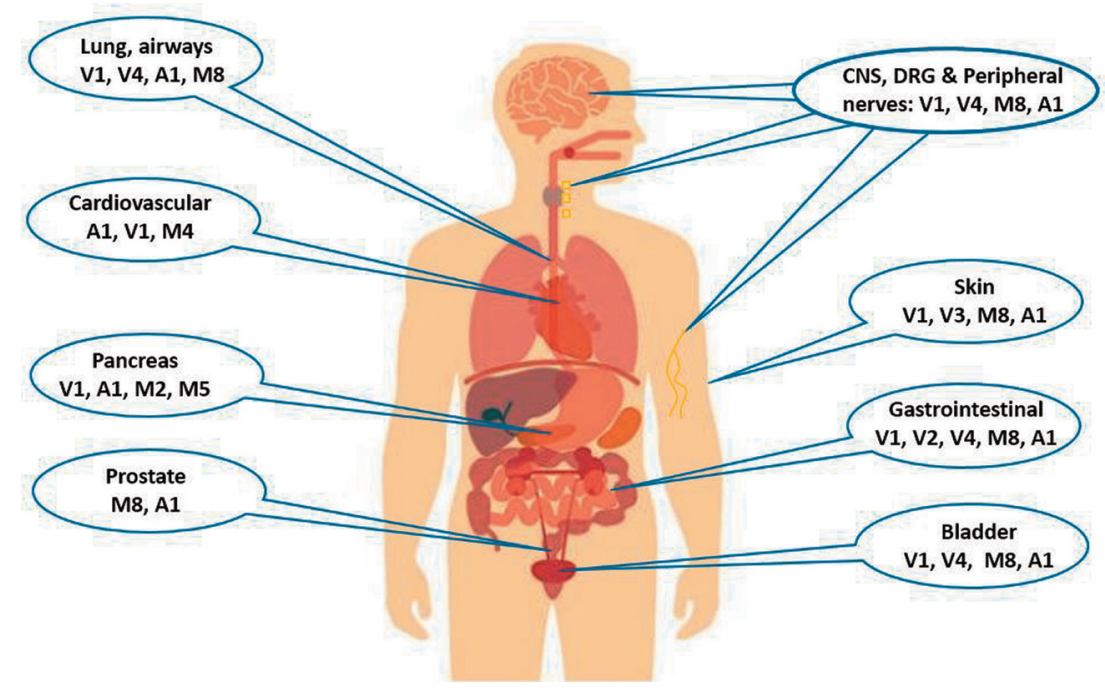

Figure 2. Schematic overview of thermo-TRP channel expression in different mammalian organs.

and hot temperatures in native and in heterologous cell systems. They exhibit temperature thresholds for activation ranging from $\sim 25^{\circ} \mathrm{C}$ for TRPV4 to $\sim 52^{\circ} \mathrm{C}$ for TRPV2.

\subsubsection{TRPV1}

The first mammalian TRPV was identified by expression cloning searching for the pharmacologically known capsaicinactivated channel [7]. Functional characterization of the cloned receptor revealed that it was gated by noxious heat with an activation threshold of $42^{\circ} \mathrm{C}$. This seminal finding provided the underlying molecular mechanism of capsaicin nocifensive effect, i.e. burning sensation [8]. Additional data showed that TRPV1 is a polymodal channel that can also be activated by extracellular acidic $\mathrm{pH}$ and membrane depolarization. Furthermore, endogenous substances such as $\mathrm{N}$-arachidonoyl dopamine, leukotriene $\mathrm{B}$, phospholipase $\mathrm{C}$ (PLC), and other natural molecules, including resiniferatoxin (RTX), piperine, gingerol, zingerone, camphor, eugenol, ethanol, and spider and jellyfish venom proteins activate TRPV1 as well [9]. Noteworthy, TRPV1 activity strongly desensitizes in the continuous presence of an activating stimulus. Receptor desensitization is a negative feedback mechanism that includes both a gating mechanism driven by channel closure and retrieval of membrane channels. Furthermore, TRPV1 tachyphylaxis is $\mathrm{Ca}^{2+}$-dependent and implies CaM-induced phosphorylation of the receptor and, for long ligand exposure, TRPV1 endocytosis, and degradation in lysosomes [10].

The complexity of TRPV1 channel cellular function is further increased due to its participation in signaling protein networks. To date, 94 proteins have been identified to interact with TRPV1 and several have been shown to modulate channel trafficking, cell localization, membrane recruitment, and signaling [11]. Indeed, $\mathrm{Ca}^{2+}$-dependent neuronal exocytosis of vesicular TRPV1 channels to the cell membrane has been proposed as an important regulatory mechanism in inflammatory and algesic conditions [12].

Expression of TRPV1 initially seemed to be restricted to small-medium sensory neurons of the dorsal root and trigeminal ganglia (DRG and TG), but it has also been reported to be expressed in a variety of neurons throughout the whole neuroaxis of the rat, from the olfactory bulb through the cortex and basal ganglia to the cerebellum [13]. Similarly, the channel is also present in non-neuronal cells in kidney, pancreas, testes, uterus, spleen, stomach, small intestine, liver, lung, bladder, skin, skeletal muscle, mast cells, macrophages, and leukocytes [14].

Currently, TRPV1 is implicated in multiple physiological and pathological processes. Physiologically, TRPV1 is involved in thermosensation, energy homeostasis, modulation of autophagy and proteasome activity, reciprocal crosstalk between the sensory nervous and immune systems. TRPV1 dysfunction has been associated with regulation of diet-induced obesity, insulin, and leptin resistance, pain, cancer, the development of severe bronchial asthma, psoriasis, itch, and inflammation [15]. The validation TRPV1 as a key therapeutic target for diverse pathologies, particularly chronic pain and pruritus, has provoked an outbreak of TRPV1-based drug discovery programs in major pharmaceutical and smaller biotechnology companies.

\subsubsection{TRPV2}

Another member of the TRPV family is TRPV2, which shares about 50\% identity with TRPV1 [16]. In contrast to TRPV1, TRPV2 is not activated by vanilloids, protons, or membrane depolarization. However, TRPV2 can be activated by high temperatures exhibiting a threshold above $52^{\circ} \mathrm{C}$ in recombinant systems, although its physiological role in temperature sensing has not been fully demonstrated, as inferred from the knockout model [17].

There are several compounds able to modulate TRPV2, however few of them are specific, and most show speciesdependent variability. 2-Aminoethoxydiphenyl borate (2-APB), probenecid, and cannabidiol (CBD) are able to activate TRPV2. Ruthenium red, SKF96365, Gadolinium (Gd), and Tranilast are channel antagonists [18]. At physiological level, TRPV2 function can be regulated by phosphoinositides and phosphorylation, as well as by post-transcriptional modifications, lipid- and protein-protein interactions [16]. In many cells, most of TRPV2 
is located in the endoplasmic reticulum and can be translocated to the plasma membrane upon stimulation with phosphatidylinositol 3-kinase-activating ligands or mechanical stress [19].

At variance with TRPV1, TRPV2 is expressed in medium- to large-diameter neurons which give rise to myelinated $A \delta$ - or $A \beta$-fibers in the DRG [16]. The widespread distribution of TRPV2 in the spinal cord suggests several physiological roles besides nociception [20]. In the forebrain, the expression of TRPV2 is the highest among various members of the TRPV family [21]. TRPV2 mRNA or immunoreactivity has also been detected in skeletal and vascular myocytes, blood cells, mainly macrophages, epithelial cells, and visceral organs including the intestine, pancreas, spleen, and bladder [16]. In addition, several studies have shown that TRPV2 is also expressed in various types of tumor cells [22].

Despite of its presence in various tissues and organs, the physiological role of TRPV2 has not been completely elucidated. Due to its stretch-dependent properties, this channel has been proposed to act as a mechanosensor and/or osmosensor [23]. TRPV2 appears involved in the regulation of intestinal motility [24], in innate and adaptive immune responses [25], and in early osteoclastogenesis [26]. TRPV2 channels expressed in cardiomyocytes could play a significant role in the regulation of intracellular $\mathrm{Ca}^{2+}$ and, therefore, in cardiac contractility [27]. In the endocrine system, TRPV2 seems to participate in the autocrine feedforward action of insulin in pancreatic $\beta$-cells [28].

TRPV2-mediated signaling pathways have profound effects on a variety of pathological processes such as cardiomyopathies and muscular dystrophy [29], diabetes and obesity [30], fibromyalgia [31], and infection diseases [32]. Several studies have demonstrated that TRPV2 exhibits an oncogenic activity in different types of cancers, controlling survival, proliferation, migration, angiogenesis, and invasion signaling pathways [22]. Hence, TRPV2 could represent a prospective prognostic marker and potential therapeutic target for novel interventions.

\subsubsection{TRPV3}

TRPV3 is a heat-sensitive ion channel that shares $43 \%$ sequence similarity with TRPV1. This channel can be activated by temperatures between $32^{\circ} \mathrm{C}$ and $39^{\circ} \mathrm{C}$ and exhibits progressively increasing responses to repeated heat stimulation [33]. TRPV3 also responds to natural compounds found in plants such as oregano, camphor, and thyme [34]. Activation of Gqcoupled receptors (GPCRs), as well as key components in the downstream signaling cascade including $\mathrm{PKC}, \mathrm{Ca}^{2+}$ calmodulin, phosphoinositides, and unsaturated fatty acids sensitize TRPV3 [35]. Other modulators include voltage, ATP, $\mathrm{Mg}^{2+}$, and intracellular acidification [36]. It has been proposed that TRPV3 forms a signaling complex with epidermal growth factor receptor (EGFR), whereby activation of this receptor results in increased TRPV3 channel activity that leads to release of transforming growth factor (TGF)- $a$ and epidermal homeostasis [37].

TRPV3 is robustly expressed in human DRG neurons, but it could not be detected in mouse DRGs. TRPV3 is abundantly present in the skin, especially in epidermal and hair follicle keratinocytes, as well as in oral and nasal epithelia [36].
Activation of TRPV3 in skin keratinocytes releases inflammatory mediators including ATP, prostaglandin (PG)E2 and interleukin-1 $\beta$ that, in turn, regulate diverse dermal functions including skin barrier formation, hair growth, wound healing, temperature sensing, as well as itch and pain perceptions [38] Hence, a properly balanced function of TRPV3 appears to be critical for all these physiological functions. Genetic mutations, gene knock-out, and selective pharmacological tools are providing insights into TRPV3 therapeutic potential. It has been demonstrated that genetic deletion of TRPV3 in mice compromises skin barrier formation and causes anomalies in hair morphogenesis [37]. Conversely, increased TRPV3 activity through gain-of-function mutations in humans causes a variety of dermatological diseases, including Olmsted syndrome [39] and palmoplantar keratodermas [40], that are accompanied by alopecia, dermal inflammation, and itch. These findings suggest a potential use of TRPV3 blockers in the treatment of skin diseases [41]. Furthermore, overexpression of TRPV3 is implicated in the development and progression of colorectal and lung cancer [42].

Of relevance to pain, TRPV3 mediated ATP release from keratinocytes has been shown to activate purinoceptors in DRG neurons [43], thereby influencing nociceptive function. Furthermore, alterations in TRPV3 receptor levels have been found in the sciatic nerve constriction pain model and in DRG neurons after traumatic injury [44]. Similarly, dysfunction of TRPV3 has been reported in diabetic neuropathy and in keratinocytes from a breast cancer patients [33], further supporting its role in pain transduction. Thus, TRPV3 antagonists may have clinical utility not only in dermatological diseases such as psoriasis and ichthyoses, but also in inflammatory pain.

\subsubsection{TRPV4}

The TRPV4 channel was one of the first channels identified as the molecular detector of osmotic changes, pressure, and shear stress in both neurons and in muscles [45]. This channel can be activated by diverse stimuli including moderate heat $\left(\sim 34^{\circ} \mathrm{C}\right)$, cell swelling, endogenous chemicals such as anandamide, arachidonic acid, and its metabolites, as well as by a growing number of exogenous chemical ligands which include natural, plant-derived compounds, phorbol esters, and synthetic ligands. Ruthenium red, gadolinium, and lanthanum have been classically used to block TRPV4 channels, although none of them is specific [46]. To date 38 proteins have been identified to interact with TRPV4 and several have been shown to modulate cellular localization and signaling [47]. Additionally, TRPV4 activity can be post-translationally enhanced via PKA- and PKC-dependent phosphorylation [48].

TRPV4 is broadly expressed in DRG, TG, and nodose ganglion (NG) neurons, bladder urothelium, kidney epithelium, vascular endothelium, inner ear, pulmonary aortic smooth muscle, skeletal muscle fibers, cardiac fibroblasts, pancreatic islets, myocytes, keratinocytes, adipocytes, and chondrocytes [49]. Due to its wide expression profile, TRPV4 is implicated in multiple pathophysiological states. TRPV4 sensitization by inflammation-triggered intracellular signaling leads to pain behavior in mice to hypo-osmotic and mechanical stimuli. In the vasculature system, TRPV4 is a regulator of vessel tone and 
is implicated in hypertension in diabetes due to endothelial dysfunction. TRPV4 is a negative regulator of epithelial and endothelial barrier function, as its function can disrupt these critical protective barriers [50]. In respiratory function, TRPV4 is involved in cystic fibrosis, ciliary beat frequency, bronchoconstriction, chronic obstructive pulmonary disease, pulmonary hypertension, acute respiratory distress syndrome, and cough [51]. Furthermore, a high number of mutations have been found in the TRPV4 gene compared to the other members of the TRP superfamily, that are causative for several human diseases, which affect the skeletal and the peripheral nervous systems, with highly variable phenotypes [46].

Modulation of TRPV4 function is a vital signaling component in a range of tissues and, therefore, development of new specific modulators may lead to novel therapeutic approaches to treat a range of disease states. In animal models, TRPV4 antagonists have provided significant therapeutic benefit to pathologies such as pain, bladder hyperactivity, cardiovascular disease, osteoarthritis, congestive heart failure, and eye disorders including glaucoma and diabetic retinopathy [52]. Regarding channel agonists, although the systemic administration results in cardiovascular collapse and death in rats, their intra-articular delivery could mitigate cartilage degradation in osteoarthritis [53]. Therapeutic potential for TRPV4 agonists has also been postulated for other diseases including polycystic kidney disease, atherosclerosis, and cancer [54].

\subsection{TRPM subfamily}

Another important family within the TRP ion channel superfamily is the transient receptor potential melastatin (TRPM) receptors, composed of eight members. They can be subdivided into four groups, based on structural homology: TRPM1 and 3, TRPM4-5, TRPM6-7, and TRPM2 and 8 [55]. Most of these plasma membrane ion channels are nonselective $\mathrm{Ca}^{2+}$ permeable (TRPM1-3 and TRPM6-8), while TRPM4/5 are $\mathrm{Ca}^{2+}$ impermeable. In addition, TRPM6-7 are $\mathrm{Mg}^{2+}$-permeable and involved in mammalian $\mathrm{Mg}^{2+}$ homeostasis [56]. TRPM members are differently expressed in many tissues, including primary sensory neurons (TRPM3, TRPM8), prostate (TRPM8), pancreatic $\beta$-cells (TRPM2, TRPM3, TRPM5), retina cells (TRPM1), kidney (TRPM6), liver, and heart (TRPM4), among others [57]. Unlike the other TRP channels, TRPM channels lack the $\mathrm{N}$-terminal ankyrin repeats.

These channels have diverse physiological functions and are implicated in different pathological processes, like inflammatory and neuropathic pain, cancer, neurodegenerative diseases, and ocular dysfunctions [58]. With the only exception of TRPM8 modulators, the above indicated therapeutic interest contrast with the low number of agonists and antagonists discovered to date for the other TRPM channels, although preclinical progress is being noticed for TRPM3 channels.

\subsubsection{TRPM8}

Transient receptor potential melastatin eight (TRPM8) channels are nonselective cationic conducting proteins, with a certain selectivity for $\mathrm{Ca}^{2+}$ permeation [59]. Initially identified as a biomarker for prostate cancer, TRPM8 receptors are highly expressed in $A \delta$ and $C$ fiber afferents of peripheral sensory neurons [60]. TRPM8 is also present in many other tissues, including lung, the cardiovascular system, the urogenital tract, and the central nervous system [61]. An appealing compendium on the distribution of TRPM8 channels, their importance in regulating different physiological processes and the clinical implications has recently been published [62]. These channels are gated by different physical and chemical stimuli, voltage, cold $\left(<28^{\circ} \mathrm{C}\right)$, osmolality, and cooling natural and synthetic compounds (menthol, eucalyptol, borneol, and icilin) [59]. Some physiological lipids (phosphoinositides), hormones (testosterone), and proteins (Pirt) have also been described as endogenous modulators of TRPM8 channels [63].

Experimental evidence implies an involvement of TRPM8 channels in cold allodynia and hyperalgesia after inflammation or nerve injury, as well as a potential implication in migraine [64]. Similarly, the aberrant expression of TRPM8 channels in different types of cancer (prostate, pancreas, breast, lung, colon, and skin) has been correlated with tumor cell progression and migration, as well as with tumor aggressiveness [65]. In addition, TRPM8 channels have also been associated with oropharyngeal dysphagia (OD), irritable bowel syndrome, chronic cough, and hypertension [66]. Therefore, the TRPM8 receptor could be contemplated as a valuable therapeutic target for the treatment of these pathologies, leading to the search of modulators by academic centers and pharmaceutical companies $[67,68]$. Both agonists and antagonists discovered to date are contributing to shed light on the role of TRPM8 channels in pathophysiological processes and may become good therapeutics.

\subsection{TRPA subfamily}

TRPA 1 is the only mammalian member of the ankyrin subfamily. It is characterized by an unusually high number of ankyrin repeats at $\mathrm{N}$-terminus (14), and up to 11 reactive cysteines available for covalent modification. TRPA 1 lacks the typical 25amino acid TRP domain in the C-terminus, although it displays a TRP-like domain [69]. The role of TRPA1 as thermosensor remains controversial [70]. Acute cold $\left({ }^{\circ} 17^{\circ} \mathrm{C}\right)$ can trigger TRPA1-mediated currents in vitro in recombinant expressed channels, although it does not appear to play a role in the control of body temperature [71] or in thermal preference in mice [72].

TRPA 1 is gated by an unusually broad variety of chemicals (allicin, carvacrol, cinnamaldehyde, gingerol, thymol, and allyl isothiocyanate) and environmental irritants (acrolein, formalin, and $\alpha, \beta$-unsaturated aldehydes), being a major contributor to chemo-nociception [69]. TRPA1 also allows cells to respond to a plethora of endogenously produced reactive molecules, particularly the reactive oxygen species (ROS) [73], and can be activated by inflammatory agents such as bradykinin and ATP through Gq-PLC-coupled receptors. TRPA1 can be blocked by nonselective compounds as ruthenium red, gentamicin, amiloride, and gadolinium. Selective inhibitors include 2-(1,3-dimethyl-2,6-dioxo-1,2,3,6-tetrahydro-7 H-purin-7-yl)$\mathrm{N}$-(4-isopropylphenyl)acetamide ( $\mathrm{HC}-03003)$ and its derivatives synthesized to improve poor pharmacokinetics properties, 4-(4-Chlorophenyl)-3-methylbut-3-en-2-one oxime (AP18), and $\quad N$-\{4-[2,4-difluoro-3-(trifluoromethyl)phenyl]-1,3-thiazol- 
2-yl\}-2-(1,3-di-methyl 2,4-dioxo-1,2,3,4-tetrahydrothieno [2,3-d] pyrimidin-5-yl) acetamide (GRC17536) [74].

TRPA1 was first identified in human fetal lung fibroblasts as a transformation-associated gene product. Later studies showed that TRPA1 was highly expressed in sensory neurons of DRG, TG, and NG, signaling this channel as a putative component for the propagation of noxious and inflammatory stimuli [75]. TRPA1 expression was also reported in various non-neuronal tissues like hair cells of the inner ear, melanocytes, vascular endothelium, heart, and lung fibroblasts, epithelial cells in small intestine, lung, bladder and pancreas, enterochromaffin cells, astrocytes, and pancreatic $\beta$-cells [69].

Human and animal data implicate TRPA 1 in a variety of pain conditions and are thought to play a major role in the pathogenesis of several disease states. Gain-of-function mutations in the human gene cause a familial episodic pain syndrome, in which debilitating upper-body pain can be triggered by stressors [76]. In rodents, neuropathic injury increases neuronal expression of TRPA1 [77]. Furthermore, genetic deletion or pharmacological inhibition of TRPA1 reduces pain behaviors in inflammatory, visceral, and neuropathic pain states [78]. There is evidence linking TRPA1 to the cold allodynia that develops following chemotherapy [79]. In animals with experimental osteoarthritis, blocking pharmacologically TRPA1 attenuated mechanical hypersensitivity in nociceptive neurons of the spinal dorsal horn [80]. The TRPA1 antagonist HC-030031 attenuated bladder overactivity in models of cyclophosphamide-induced cystitis and spinal cord injury [81]. In respiratory disorders, TRPA1 expressed by lung fibroblasts might play a role in the pathogenesis of asthma and Chronic Obstructive Pulmonary Disease (COPD) [82]. Furthermore, TRPA 1 has also been implicated in migraine, dental pain, and pain secondary to diabetic neuropathy [69].

These data have prompted significant interest in TRPA1 as a therapeutic target. However, a major drawback for pharmaceutical development is the substantial species-dependent differences in TRPA1 function both in response to pharmacological compounds and to thermal stimuli [83]. This appears related to the genetic distance between humans and mice orthologs compared to other ion channels. Recently, a human TRPA1-specific pain model has been published, this could help to bridge the translational gap between pre-clinical and clinical research [84].

\section{ThermoTRP channels modulators in clinical trials}

\subsection{TRPV subfamily}

\subsubsection{TRPV1}

Following the seminal discovery of TRPV1 and its implication in pain, pharmaceutical and academic laboratories have spent more than 20 years researching this protein as a therapeutic target for analgesia. From TRPV1 cloning, it took around 7 years until AMGEN brought the first antagonist, $\mathrm{N}$-[4-[[6-[4-(Trifluoromethyl)phenyl]-4-pyrimidinyl]oxy]-2-benzothiazolyl]acetamide (AMG-517), to Phase I clinical trials [85]. Since then, a large number of TRPV1 antagonists including $\mathrm{N}$-(2-bromophenyl)- $\mathrm{N}^{\prime}$-[(3 R)-1-[5-(trifluoromethyl)-2-pyridinyl]3-pyrrolidinyl]-urea (SB-705,498, GlaxoSmithKline), 5'-chloro-7'methyl-1'-[[3-(trifluoromethyl)phenyl]methyl]spiro [imidazolidine-5,3'-indole]-2,2',4-trione (AZD-1386) and NEO-6860 (AstraZeneca), and 2-Propenamide, N-[(1 R)-1-[3,5-difluoro4-[(methylsulfonyl)amino]phenyl]ethyl]-3-[2-propyl-6-(trifluoromethyl)-3-pyridinyl]-, (2E)- (PAC-14,028, Amorepacific Corporation) have been advanced to Phase I and II clinical studies for indications related to pain $[6,15]$ (Table 1).

The enthusiasm, however, was soon tempered by unforeseen adverse effects. The first generation of TRPV1 inhibitors, such as AMG-517, caused elevated body temperature in a Phase I clinical trial [3] and reduced their ability to sense noxious heat [86]. Similarly, other highly potent first generation of TRPV1 antagonists also produced hyperthermia. A remarkable exception was the modality-specific modulator

Table 1. Current clinical phase status of TRPV-targeted therapies.

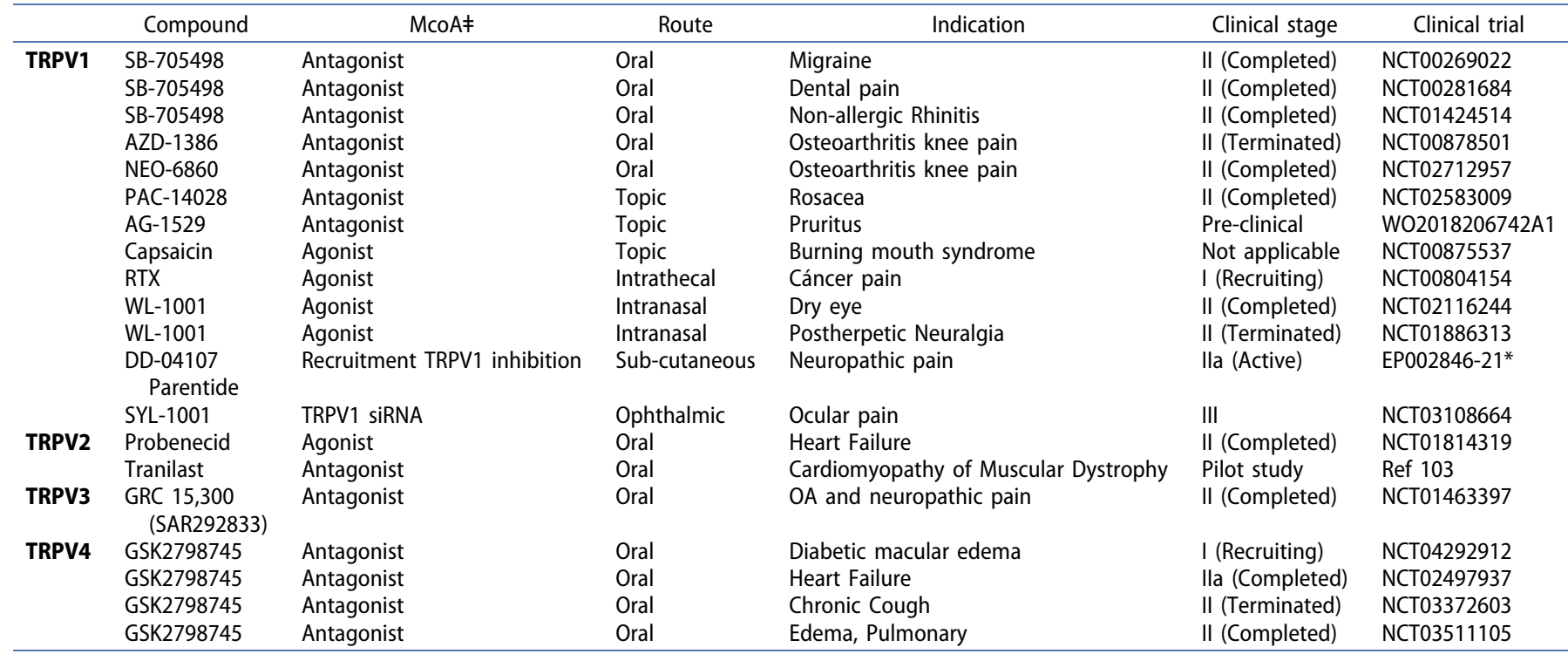

* EudraCT Number. https://www.clinicaltrialsregister.eu/ctr-search/search?query=parentide

₹ McoA: Mechanism of action 
NEO-6860 that did not affect pH and heat evoked TRPV1 gating and did not alter body temperature in humans, suggesting that hyperthermia was associated with modality-based block of TRPV1 gating. This compound is currently being developed for osteoarthritic pain [87]. However, limited data are available on the outcome of these clinical studies, and consequently, the analgesic potential of NEO-6860 is still unknown.

To avoid the systemic side effects of TRPV1 antagonists, topical formulations of these compounds are being developed for treating peripheral neuropathies. Examples are formulations of AMG-9810 and PAC-14,028. However, long topical use of AMG-9810 has been reported to promote tumor development through the EGFR/Akt/mTOR signaling pathway in pre-clinical murine models [88]. However, recent evidence has challenged the potential peripheral carcinogenic effects of TRPV1 antagonists [89]. Thus far, only PAC-14,028 has progressed to Phase III trials for topical treatment of pediatric atopic dermatitis.

On the other hand, agonists that are able to desensitize TRPV1 have been used for decades as analgesics. The bestknown example is capsaicin containing creams (e.g. Zostrix, $0.075 \%$ ) for the treatment of chronic painful conditions such as diabetic neuropathy and pruritus [90]. In spite of its popularity, some controlled clinical studies questioned that these creams had significantly greater analgesic potency than placebo [91]. It should be noted that this controversy could be related by the burning effects displayed by topical capsaicin that reduces its use by patients.

To increase the exposure of cutaneous nerve endings to capsaicin, occlusive patches (NGX-4010, Qutenza) and liquid formulations (NGX-1998, 20\% capsaicin) were developed by Neuroges-X. Currently, another injectable capsaicin derivative, CNTX-4975, development by Anesiva, is in Phase III clinical trials for the treatment of osteoarthritis knee pain (NCT03660943). Winston Laboratories is developing WL-1001, an intranasal civamide (cis-capsaicin). This compound is currently in Phase III trials for cluster headache and osteoarthritis knee pain, and in Phase II for dry eye and postherpetic neuralgia (Table 1). The potent agonist Resiniferatoxin (RTX) is undergoing clinical trials as a 'molecular scalpel' to achieve permanent analgesia in patients with intractable cancer pain [92], but data have not been yet released.

However, despite its good therapeutic action, the burning sensation associated with TRPV1 agonists due to channel activation decreases patient adherence to the treatment [93] and often requires the use of local anesthetics during administration. In addition, some reports suggest that their longterm dermal accumulation due to their poor elimination, along with UV exposure, may induce skin carcinogenesis [94].

Given the important role of TRPV1 in pain transduction and the side effects exhibited by known TRPV1 modulators, novel strategies to modulate its dysfunctional activity are particularly eagerly pursued. An interesting approach is the development of TRPV1 antagonists that can be dermally deactivated in a predictable and controlled way after having exerted their biological activity, the so-called soft drugs [95]. This strategy has been used in the design of capsaicinoid-based molecules that undergo dermal deactivation, thus preventing their longterm dermal accumulation [96]. Compound 2-((4-hydroxy2-iodo-5-methoxybenzyl) amino)-2-oxoethyl dodecanoate (AG1529, AntalGenics) is currently in pre-clinical trials with the expectation to enter in a Phase 1/lla clinical trial in the first quarter of 2021 as a topical anti-pruritic and antiinflammatory medication.

Another original analgesic strategy is to inhibit proinflammatory recruitment of TRPV1 to the neuronal terminals. This process is mediated by the regulated exocytosis of a vesicular population of channels ready to go in response to an inflammatory or algesic insult. The therapeutic strategy considers modulating the exocytotic incorporation of vesicles containing TRPV1 to the neuronal membrane using a peptide that inhibits neuronal exocytosis. In particular, compound DD04107, a small peptide patterned after the SNAP25 protein of the neuronal SNARE complex, exhibited important and long-lasting anti-nociceptive activity in models of chronic neuropathic and inflammatory pain [97]. Intriguingly, DD04107 therapeutic activity was largely devoid of secondary effects, including the hyperthermia, implying a promising therapeutic index. Indeed, this compound is currently in Phase II clinical trials to treat chronic post-surgical pain.

A different approach to inhibit TRPV1 has been developed by Sylentis for the treatment of pain in dry eye disease, based on the use of a small interfering RNA, SYL-1001, to silence the TRPV1 expression in the ocular system [98]. Currently, SYL1001 is in Phase III for the treatment of ocular pain in patients with dry eye disease.

Taking together, TRPV1 is still considered a pivotal therapeutic target for treating a plethora of pathological processes. Accordingly, the interest of pharmaceutical and biotech companies in discovering channel modulators remains high, addressing it from different approaches which may lead in the future to the approval of the first TRPV1-based drug.

\subsubsection{TRPV2}

Despite the potential therapeutic interest of TRPV2 modulation, thus far, only two-channel modulators have shown promising pharmacological results, namely Probenecid and Tranilast as activator and inhibitor respectively (Table 1). Probenecid acts as a TRPV2 agonist and has recently become the subject of study as an investigational therapy for the treatment of cardiomyopathy due to its positive inotropic effects. Probenecid is an FDA approved drug for the treatment of gout and hyperuricemia and has been used safely in humans for decades for different indications. A Phase II clinical assay (NCT01814319) has shown that Probenecid improves cardiac function in patients with heart failure with no significant adverse effects after 1-week treatment. This therapeutic action is presumably exerted through TRPV2 activation [99]. Studies evaluating long-term safety are needed to become a new therapeutic option for patients with heart failure. A Phase IV clinical assay (NCT03965351) is ongoing to determine if Probenecid improves magnetic resonance (MRI) parameters of systolic and/or diastolic dysfunction as well as associated symptoms in patients with a Fontan circulation. 
Tranilast has been widely used as an anti-allergic drug [100]. In a pilot study, Tranilast was found to have a protective effect against cardiomyopathy provoked by muscular dystrophy [29]. This study set the stage for the use of TRPV2 inhibition as a new therapeutic strategy for muscle dysgenesis, such as cardiomyopathy and muscular dystrophy [101]. Additionally, TRPV2 is involved in signaling pathways responsible for cancer growth/proliferation, migration/invasion, and apoptosis [22], thus representing a novel promising pharmacologic target especially in the management of aggressive cancers. In this regard, Tranilast, was found to be effective in reducing proliferation in leukemia cells and cancer-associated fibroblasts in vitro. As a consequence, it is now considered a potential candidate for further pre-clinical studies alone or in combination regimens as anti-cancer drug [102].

\subsubsection{TRPV3}

Despite its potential therapeutic interest, the number of patents describing TRPV3 modulators has been modest compared to other TRPV family members. Hydra Biosciences and Glenmark Pharmaceuticals have disclosed discovery programs for this channel [41]. Up to date, there are no known clinical trials ongoing with any of their compounds or other TRPV3 modulators.

Hydra Biosciences was the first company to disclose the efficacy of their TRPV3 antagonists in vivo [103]. In 2007, they entered into a collaboration with Pfizer to develop TRPV3 antagonists for pain. The company web page currently reports an active TRPV3 program directed toward dermatological disorders, but we could not find clinical development reports disclosing results.

Glenmark also patented a series of potent and selective TRPV3 receptor antagonists [104]. In 2010 Glenmark entered into an out-licensing agreement with Sanofi Aventis. Subsequently, the lead molecule (GRC15300) progressed into clinical trials for the treatment of osteoarthritic and neuropathic pain (Table 1). However, this compound did not meet the primary endpoint in a Phase II proof-of-concept trial. Glenmark has not reported further developments in this field. Despite these clinical failures, it is expected that TRPV3 antagonists will remain interesting as pain and dermal therapeutics, because of the demonstrated role of this channel in pain transduction and cutaneous inflammatory signaling.

Interestingly, there are also several patents indicating the utility of TRPV3 agonists. One describes derivatives of incensole acetate as antidepressants [105]. Another suggests TRPV3 agonists for the treatment of TRPV3-associated skin conditions such as acne, psoriasis, dermatitis, and wound healing, and describes a TRPV3 agonist activity of peptides derived from soricidin [106]. To our knowledge, none of these compounds has reached clinical development. Nonetheless, given the concerns with the side effects associated when targeting other TRP family members, such as TRPV1, including body temperature changes and altered thermal sensitivity, there may be an opportunity to develop topical treatments that modulate TRPV3 activity considering its expression and function in the skin.

\subsubsection{TRPV4}

In recent years, significant progress has been made in TRPV4 drug discovery programs. Thus far, compounds from at least nine different chemical scaffolds have shown pharmacological activity in various animal models [107]. The first potent TRPV4 antagonist, GSK2798745, developed and patented by GlaxoSmithKline (GSK), has been investigated in four separate early phase clinical trials (Table 1). A Phase lla crossover study in adults with heart failure was completed by August 2017 (NCT02497937). Notably, the results indicated that GSK2798745 improved pulmonary function, respiration, and gas exchange, as well as sleep-disordered breathing in patients with heart failure. However, GSK terminated the development of this product for unspecified reasons.

In 2017, another clinical trial started for the treatment of chronic cough, NCT03372603, but the study terminated in 2019 due to the lack of effect. The clinical trial initiated in 2018, to study the effect of GSK2798745 on alveolar barrier disruption in a segmental lipopolysaccharide challenge model, was terminated in February 2020 due to low probability of achieving a positive outcome on the primary endpoint (NCT03511105). Recently, another clinical trial has been started using GSK2798745 for the treatment of diabetic maculae edema (DME). NCT04292912 is currently in the recruitment stage.

Research aimed at discovering TRPV4 modulators as therapeutics has increased considerably in recent years. TRPV4 has proven to be a highly druggable target, with numerous chemotypes providing potent ligands with oral bioavailability and other drug-like properties. Thus, further investigation of TRPV4 inhibition in humans is warranted to ascertain its therapeutic benefit.

\subsection{TRPM subfamily}

Research on suitable molecules for the modulation of most TRPM channels is still in its infancy, except for TRPM8 receptors. Therefore, this section only contemplates the clinical trials related to this TRP channel.

In recent years, pharmaceutical and biotech companies have been interested in developing TRPM8 modulators. This has resulted in a plethora of agonist and antagonist families $[66,68]$. However, it is surprising that only a few of these compounds have reached clinical development. In contrast, topically applied menthol has been implicated in the reduction of neurogenic inflammation, and mechanical- and heatinduced hyperalgesia [108], suggesting the potential of TRPM8 agonists as topical drugs.

Menthol has been profusely used in $>200$ clinical studies for cosmetic and medical applications, most of them related to pain (Table 2). Different menthol concentrations, from $3.5 \%$ to $40 \%$, topically applied have entered into clinical trials to treat diverse painful conditions, from Carpal tunnel syndrome to chemotherapy-induced peripheral neuropathy. Thus far, only positive results have been published for two of them. One is related to topical application of 3.5\% menthol to treat pain symptoms of patients with carpal tunnel syndrome (NCT01716767). The observed reduction of pain intensity in 
Table 2. Current clinical phase status of TRPM8-targeted therapies.

\begin{tabular}{|c|c|c|c|c|c|}
\hline Compound & McoAf & Route & Indication & Clinical stage & Clinical trial \\
\hline Menthol $(3,5 \%)$ & Agonist & Topical & Carpal tunnel syndrome & NA (Completed) & NCT01716767 \\
\hline Menthol (4\%) & Agonist & Topical & Mechanical Neck pain & NA (Completed) & NCT03012503 \\
\hline Menthol (4\%) & Agonist & Topical & Knee osteoarthritis & II (NYR) & NCT04351594 \\
\hline Menthol (5\%) & Agonist & Topical & Pain after photodynamic therapy & IV (Prospective) & NCT02984072 \\
\hline Menthol (6\%) & Agonist & Topical & Migraine & NA (Completed) & NCT01687101 \\
\hline Menthol (7\%) & Agonist & Topical & Chemotherapy-induced peripheral neuropathy & II (Recruiting) & NCT01855607 \\
\hline Menthol (40\%) & Agonist & Topical & Pain, after TRPA1 agonist & NA (Completed) & NCT02653703 \\
\hline Menthol & Agonist & Oral & Oropharyngeal dysphagia & II (Completed) & NCT03050957 \\
\hline D-3263 & Agonist & Oral & Cancer & I (Completed) & NCT00839631 \\
\hline AVX-012 & Agonist & Ophthalmic & Dry eye & I/II (Recruiting) & NCT03162094 \\
\hline PF-05105679 & Antagonist & Oral & Pain & I (Completed) & NCT01393652 \\
\hline AMG 333 & Antagonist & Oral & Migraine & I (Completed) & NCT01953341 \\
\hline
\end{tabular}

NA: Not applicable

NYR: not yet recruiting

₹ McoA: Mechanism of action

arm/hand after menthol treatment could constitute an effective non-systemic alternative to regular analgesics. The other clinical assay was based on the application of $6 \%$ menthol behind the ears and to the occipital region of the neck to treat migraine (NCT01687101). In this study, patients showed a significant improvement in headache intensity, suggesting that could be an effective treatment for acute migraine attacks.

Additionally, an oral formulation of menthol has also entered into clinical assays to evaluate the possible effect of oral menthol (bolus) on the swallow response in patients with Oropharyngeal dysphagia. No conclusions on this study have been published to date.

Another agonist, D3263, has entered into Phase I clinical trials to treat patients with solid tumors, due to the preliminary beneficial effects observed in patients with advanced prostate cancer [109]. A possible application to treat benign prostatic hyperplasia was also indicated. However, this study seems to be discontinued for unknown reasons. Based on the involvement of TRPM8 in the pathophysiology of dry eye disease [110], an agonist of this channel, AVX-012 (Avizorex Pharma), has successfully concluded Phase I and lla clinical trials to diminish the discomfort sensations elicited by ocular surface dryness. This compound has been acquired by Aerie Pharmaceuticals that will continue with the clinical development.

TRPM8 antagonists have also entered into clinical trials. Pfizer progressed a selective TRPM8 antagonist, 3-[[[( $11 R)$ 1-(4-fluorophenyl)ethyl](3 quinolinylcarbonyl)amino] methyl] benzoic acid (PF-05105679), to Phase I clinical trials after observing that was able to inhibit pain in the cold pressure test. Unfortunately, after completing Phase I, unwanted side effects were observed that precluded further clinical progress [111]. AMGEN performed a Phase I clinical study to determine the safety, tolerability, and pharmacokinetic profile of the TRPM8 antagonist 6-[[(S)-(3-fluoro-2-pyridinyl)[3-fluoro-4-(trifluoromethoxy)phenyl]methyl]amino]carbonyl]-3-pyridinecarboxylic acid (AMG 333) in both healthy subjects and patients with migraine. The results obtained evidenced some adverse effects including feeling hot, paresthesia, dysesthesia, and dysgeusia. Therefore, despite the good pharmacokinetic properties and toxicological profile [112], the final decision was to discontinue clinical development of this compound for migraine prophylaxis.

In summary, from the different clinical trials with TRPM8 modulators, it can be concluded that topical administration of TRPM8 agonists could serve to relief several types of pain (inflammatory, mechanical, peripheral neuropathies, migraine), while oral treatments with either agonists or antagonists resulted in important secondary effects that, for the moment, did not allow their progression into the clinic.

\subsection{TRPA subfamily}

TRPA1 is a well-validated pharmacological target in therapeutic areas of high unmet medical needs such as pain and respiratory disorders [69]. At least four companies (Cubist, Glenmark, Hydra Biosciences, and Orion Pharmaceuticals) initiated Phase I trials with TRPA1 antagonists (see Table 3). The first efficacy data in humans were disclosed by Glenmark. They reported positive results for GC17536 in a Phase lla trial in painful diabetic peripheral neuropathy. This proof-ofconcept study showed a statistically significant and clinically relevant response in a subgroup of patients with moderate to severe diabetic neuropathic pain.

Cubist Pharmaceuticals and Hydra Biosciences announced a joint Phase I clinical trial with TRPA1 antagonist CB-625 for acute surgical pain. Unfortunately, the assay was discontinued

Table 3. Current clinical phase status of TRPA1-targeted therapies.

\begin{tabular}{|c|c|c|c|c|c|}
\hline Compound & McoAf & Route & indication & Clinical stage & Clinical trial \\
\hline GRC17536 & Antagonist & Oral & Diabetic peripheral neuropathy & II (Discontinued) & NCT01726413 \\
\hline CB-625 & Antagonist & Oral & Acute surgical pain & I (Discontinued) & No active clinical trials \\
\hline$H X-100$ & Antagonist & Oral & $\begin{array}{l}\text { Diabetic peripheral neuropathy } \\
\text { And allergic asthma }\end{array}$ & I (Discontinued) & No active clinical trials \\
\hline ODM-108 & NAM & Oral & Neuropathic pain & I (Discontinued) & NCT02432664 \\
\hline
\end{tabular}

NAM: negative allosteric modulator

₹ McoA: Mechanism of action 
due to safety concerns resulting from the drug's low solubility [74].

ODM-108 is a potent TRPA1 antagonist developed by Orion Pharmaceuticals to treat neuropathic pain. This drug reached Phase I (NCT02432664), but the study was terminated due to complex pharmacodynamic properties. However, there were no safety concerns at the doses administered.

Health Canada approved a Clinical Trial Application (CTA) from Hydra biosciences to begin a Phase I study with HX-100 to treat painful diabetic neuropathy. In 2018, Eli Lilly acquired the TRPA1 program from Hydra Biosciences and continued with the Phase I development of $\mathrm{HX}-100$ for neuropathic pain. This program is currently ongoing in Canada (PO).

Despite significant TRPA1-directed medicinal chemistry efforts across the pharmaceutical industry in the past years, most channel modulators were discontinued, due mainly to low oral bioavailability. Thus, a key issue in the progression of TRPA1 clinical development programs is the identification of high-quality, orally bioavailable compounds. Interestingly, although a limited number of compounds have so far progressed to the clinic, significant adverse events have not been reported. This suggests that, contrary to TRPV1 modulation, TRPA1 antagonism is not associated with significant safety issues. On the other hand, considering that TRPA1 is thought to be a detector of harmful environmental irritants, the extent to which TRPA1 antagonists may lead to deficiencies in the detection of noxious substances by humans should be considered during any clinical development program for TRPA1.

\section{Conclusion}

After the seminal discovery of the first thermosensor in mammals, TRPV1, the thermoTRP family has increased over the years and its involvement in inflammation and pain has validated them as therapeutic targets thrusting intense drug discovery programs in both the academy and the industry. Progress in thermoTRP pharmacology was fast, having the first clinical candidate in less than 7 years from the therapeutic validation of TRPV1 [85]. Since then, antagonists targeting other thermoTRPVs, as well as TRPA 1 and TRPM 8 have been discovered. Concurrently, exciting new discoveries have expanded the therapeutic potential of drugs targeting thermoTRP channels by validating their contribution to the pathophysiology of several disease areas, including respiratory diseases (cough, COPD, and asthma), cardiovascular, bladder, metabolic (including obesity and diabetes), skin, neurological disorders, and cancer.

Despite the clinical relevance of thermoTRPs as pivotal therapeutic targets for treating human disorders, their progress into the clinics has been tampered by poor clinical outcomes due to either safety concerns or limited pharmacological efficacy in humans. These issues have questioned the translational value of current in vitro and in vivo pre-clinical models based on rodents and put some pressure in the development of more translational models that thrust the clinical development of thermoTRP modulators. In our review, we have also highlighted the need to further our understanding of the physiological role of thermoTRPs at both levels, their activation mode and tissue expression. The use of thermoTRP modulators that indiscriminately block all modes of channel gating and/or their function in all tissues has resulted in the development of pharmacological tools with unacceptable therapeutic index. Nonetheless, it should be stated that these initial clinical failures have provided valuable information for the design of new a generation of thermoTRP modulators with much higher therapeutic value that along with novel pharmacological strategies, should pave their way to the clinics in the next decade.

\section{Expert Opinion}

As mentioned, clinical modulation of thermoTRPs has been more challenging than previously anticipated. This field has driven from an initial enthusiastic expectation, particularly in the field of chronic inflammation and pain, to a frustrated sensation upon the first clinical failures. Fortunately, the continuous progress on the role of these channels in different tissues and physiological functions, along with their contribution to the etiology of human diseases, and the knowledge of their atomic structure is helping to revisit the pharmacological approaches by designing more thoughtful compounds directed to modulate malfunctional channels. Furthermore, most of our knowledge about the role of these channels in different pathologies was obtained in knockout mice and rodent models of human disease. Sadly, it is becoming increasingly clear that these models do not flawlessly mimic the human disease as exemplified by the lack of analgesic effect of the TRPV1 antagonist AZD1386 in clinical trials enrolling patients with osteoarthritic pain [113], or the lack of efficacy of the TRPV4 antagonist GSK2798745 in the treatment of chronic cough (NCT03372603).

In our opinion, there are important pre-clinical aspects that need to be refined to ensure the clinical development of thermoTRP channel modulators as useful therapeutics to treat a plethora of human diseases. Among them, the more important issues to be addressed in the near future are:

1.To increase the clinical translation of pre-clinical models of human diseases. This appears essential to increment the efficacy of drug candidates in Phase II and III trials. Initial promising expectations of animal models of human disease have been disappointing because of their poor clinical translation. We believe that this is probably due to two main factors: (i) species differences in the pharmacology of thermoTRP channel that impact the therapeutic efficacy of drug candidates; and, (ii) lead compounds have been traditionally assayed in in-bred, genetically identical rodents. In addition, these assays have been performed in males to avoid the hormonal variability of females. This highly controlled and homogeneous animal cohort provides results that are difficult to translate to the highly heterogeneous human cohorts in clinical trials. Thus, we consider that surpassing these limitations requires the development and validation of humanbased pre-clinical models that allow testing of compound efficacy and potency in disease-relevant cells. We believe that current advances in the development of in vitro humanbased organoids will furnish pre-clinical models that will enhance the clinical development of thermoTRP modulators. In this regard, the progress in cellular reprogramming of iPSC 
derived from fibroblasts to sensory neurons, along with their in vitro culturing in microfluidic chambers that allow compartmentation of soma and axons represent a significant step in this direction. Implementation of human-based organoids obtained with patient-derived cells will provide a more realistic pre-clinical model for early validation of thermoTRP drug candidates.

2.To reduce unwanted side effects seen in Phase I and II clinical trials. Although some potential side effects are detected in pre-clinical assays (for instance, cardiotoxicity due to hERG blockade), there are others that have escaped attention, particularly body temperature and sensorial effects $[3,86]$. Current pre-clinical protocols are undoubtedly aware of these potential side effects and have designed specific tests for early detection, which prevents the progress of candidates into expensive clinical trials. Side effects arise from either offtarget and/or off-tissue interactions of drug candidates. We believe that off-target effects can be reduced improving the interaction of compounds with dysfunctional channels, which requires a better understanding of the conformational state of these proteins, particularly because of their multimodal activation. The recent progress of cryo-electromicroscopy, along with in silico methods, should critically contribute to refine the discovery of either modality-specific modulators targeting preferentially one activation mode (chemical or physical), or activity-dependent compounds preferentially binding to overactivated, algesically sensitized receptors (i.e. uncompetitive antagonists).

A complementary therapeutic approach could be regulating thermoTRP neuronal expression induced by proalgesic and inflammatory mediators, particularly in chronic conditions. This approach preserves the physiological activity of channel as only modulates the amount of surfaceexpressed channel in injured tissues. The anti-nociceptive activity of DD04107 (Parentide ${ }^{\oplus}$, BCN Peptides) in chronic inflammatory and neuropathic pain exemplifies this strategy and lend support to $\mathrm{Ca}^{2+}$-dependent-exocytosis of thermoTRPs as an important cellular mechanism involved in pain transduction that may lead to better therapies [97]. Indeed, a Phase II clinical trial with Parentide ${ }^{\circledR}$ is already ongoing to treat neuropathic pain. A note of caution should be raised as potent inhibition of neuronal exocytosis may lead to severe toxic effects as those shown by botulinum neurotoxins. Intriguingly, Parentide ${ }^{\oplus}$ exhibited a good safety profile at therapeutically relevant doses.

To control off-tissue side effects, we believe that local delivery either directly on the affected tissue or systemically using a delivery system is the most appropriate strategy. Direct local or topical application of thermoTRP modulators avoids the unwanted side effects associated with systemic distribution. As a case in point, the skin is an accessible tissue for topical administration of drugs to treat thermoTRP-mediated cutaneous disease states, including dermatitis, psoriasis, chronic pruritus, peripheral neuropathies, and skin cancer. Nonetheless, caution should also be exerted as dermal accumulation of these compounds may lead also to long-term toxicity that could be potentiated by UV exposure, particularly dermal carcinogenesis. Thus, the use of alternative strategies that facilitate the dermal elimination of drugs such as the use of soft drugs seems a worth strategy to be explored for limiting the cutaneous residence time. Dermal soft drugs are a kind of compounds that suffer metabolic deactivation by dermal esterases giving rise to enzymatic products that are readily eliminated. A proof of concept for this strategy has been used to design agonists and antagonists of TRPV1 for treating skin conditions. Compound AG1529 (AntalGenics) is a lead compound for psoriatic pruritus. Other examples of local delivery apply to the treatment of gynecological and ophthalmological disorders, as well as for the treatment of osteoarthritis.

We believe that the use of novel, nanotechnology-based tissue oriented, smart delivery systems may help to limit the side effects of promising drug candidates. The progress on innovative polymeric materials to obtain nanoparticles and nanofibers that can be loaded with a drug or a combination of drugs, along with tissue-specific tags, represents a valuable strategy to systemically deliver thermoTRP drugs to dysfunctional tissues minimizing the interaction with healthy organs.

The development of clinically useful thermoTRP modulators remains with high expectations as therapeutics for human diseases that need medical treatment. Although their development into useful medicines has faced problems that precluded their clinical progress, new technological and pharmaceutical developments aimed at approaching pre-clinical settings to clinically relevant responses will result in the future approval of thermoTRPbased drugs. In addition, the current progress in personalized medical strategies based on the use of prognostic biomarkers that help in the stratification of patients for treatment will increase the therapeutic use of drugs targeting dysfunctional thermoTRP channels.

\section{Acknowledgments}

We thank the support of AEI (grants RTI2018-097189-B-C2-1and RTC-20176507-1), with FEDER funds from EU "Una manera de hacer Europa" and UMH (grant PAR-2019).

\section{Funding}

This study was funded by Grants from AEI (MICIU) RTI2018-097189-B-C2-1 (to AFM and AFC), RTI2018-097189-C2-2 (to RGM), RTC-2017-6507-1 (to AFM), with FEDER funds from EU "Una manera de hacer Europa" and grants from the UMH, PAR2019 (to AFM) and CSIC, 201980 E030 (to RGM).

\section{Declaration of interest}

AFC, AFM, and GFB are founders of the spin-off Antalgenics SL. AFM and AFC are inventors of WO20188206742 (A1) and WO2012032209 (A3) patents, and AFM is inventor of WO2008049945 (A1) and WO2010009892 (A2). The authors have no other competing interest to declare. The authors have no other relevant affiliations or financial involvement with any organization or entity with a financial interest in or financial conflict with the subject matter or materials discussed in the manuscript. This includes employment, consultancies, honoraria, stock ownership or options, expert testimony, grants or patents received or pending, or royalties. 


\section{Reviewer disclosures}

Peer reviewers on this manuscript have no relevant financial or other relationships to disclose.

\section{References}

Papers of special note have been highlighted as either of interest $(\cdot)$ or of considerable interest $(\cdot \bullet)$ to readers.

1. Kaleta M, Palmer C. TRP channels in yeast. Adv Exp Med Biol. 2011;704:315-321.

2. Yuan P. Structural biology of thermoTRPV channels. Cell Calcium. 2019;84:120.

3. Moran MM. TRP channels as potential drug targets. Annu Rev Pharmacol Toxicol. 2018;58:309-330.

4. Himmel NJ, Cox DN. Transient receptor potential channels: current perspectives on evolution, structure, function and nomenclature. Proc Biol Sci. 2020;287:1-9.

- A very comprehensive review on general properties and pharmacological significance of thermoTRPs.

5. Valente P, García-Sanz N, Gomis A, et al. Identification of molecular determinants of channel gating in the transient receptor potential box of vanilloid receptor I. Faseb J. 2008;22:3298-3309.

6. Ferrer-Montiel A, Fernández-Carvajal A, Planells-Cases R, et al. Advances in modulating thermosensory TRP channels. Expert Opin Ther Pat. 2012;22:999-1017.

- A key reference providing an overview of the recent development regarding thermoTRP channel modulators.

7. Caterina MJ, Pang Z. TRP channels in skin biology and pathophysiology. Pharmaceuticals (Basel). 2016;9:77-104.

- A seminal paper that describes an elegant strategy to clone the first thermoTRP channel, TRPV1.

8. Tominaga M, Caterina MJ, Malmberg AB, et al. The cloned capsaicin receptor integrates multiple pain-producing stimuli. Neuron. 1998;21:531-543.

9. Jeon S, Caterina MJ. Molecular basis of peripheral innocuous warmth sensitivity. Handb Clin Neurol. 2018;156:69-82.

10. Sanz-Salvador L, Andrés-Borderia A, Ferrer-Montiel A, et al. Agonistand Ca2+-dependent desensitization of TRPV1 channel targets the receptor to lysosomes for degradation. J Biol Chem. 2012;287:19462-19471.

11. TRIP Database-TRPV1; [cited 2020 Sep 10. Available from: http:// trpchannel.org/summaries/TRPV1

12. Mathivanan S, Devesa I, Changeux J-P, et al. Bradykinin induces TRPV1 exocytotic recruitment in peptidergic nociceptors. Front Pharmacol. 2016;7:1-12.

13. Sawamura S, Shirakawa $H$, Nakagawa T, et al. Neurobiology of TRP channels. Boca Raton (FL): CRC Press/Taylor \& Francis; 2017.

14. Bevan S, Quallo T, Andersson DA. TRPV1. Handb Exp Pharmacol. 2014;222:207-245.

15. Kaneko Y, Szallasi A. Transient receptor potential (TRP) channels: a clinical perspective. Br J Pharmacol. 2014;171:2474-2507.

16. Kojima I, Nagasawa M. TRPV2. Handb Exp Pharmacol. 2014;222:247-272.

17. Park U, Vastani N, Guan Y, et al. TRP vanilloid 2 knock-out mice are susceptible to perinatal lethality but display normal thermal and mechanical nociception. J Neurosic. 2011;31:11425-11436.

18. Perálvarez-Marín A, Doñate-Macian $P$, Gaudet R. What do we know about the transient receptor potential vanilloid 2 (TRPV2) ion channel? Febs J. 2013;280:5471-5487.

19. Doñate-Macián P, Enrich-Bengoa J, Dégano IR, et al. Trafficking of stretch-regulated TRPV2 and TRPV4 channels inferred through interactomics. Biomolecules. 2019;9:1-13.

20. Shibasaki K. Physiological significance of TRPV2 as a mechanosensor, thermosensor and lipid sensor. J Physiol Sci. 2016;66:359-365.

21. Nedungadi TP, Dutta $M$, Bathina $C S$, et al. Expression and distribution of TRPV2 in rat brain. Exp Neurol. 2012;237:223-237.
22. Santoni G, Amantini C, Maggi F, et al. The TRPV2 cation channels: from urothelial cancer invasiveness to glioblastoma multiforme interactome signature. Lab Invest. 2020;100:186-198.

23. Aguettaz E, Bois $P$, Cognard C, et al. Stretch-activated TRPV2 channels: role in mediating cardiopathies. Prog Biophys Mol Biol. 2017;130:273-280.

24. Mihara $\mathrm{H}$, Boudaka $A$, Shibasaki $\mathrm{K}$, et al. Involvement of TRPV2 activation in intestinal movement through nitric oxide production in mice. J Neurosci. 2010;30:16536-16544.

25. Santoni G, Farfariello V, Liberati $S$, et al. The role of transient receptor potential vanilloid type-2 ion channels in innate and adaptive immune responses. Front Immunol. 2013;4:1-9.

26. Kajiya $H$, Okamoto $F$, Nemoto $T$, et al. RANKL-induced TRPV2 expression regulates osteoclastogenesis via calcium oscillations. Cell Calcium. 2010;48:260-269.

27. Koch SE, Gao X, Haar L, et al. Probenecid: novel use as a non-injurious positive inotrope acting via cardiac TRPV2 stimulation. J Mol Cell Cardiol. 2012;53:134-144.

28. Uchida K, Dezaki K, Yoneshiro T, et al. Involvement of thermosensitive TRP channels in energy metabolism. J Physiol Sci. 2017;67:549-560.

29. Matsumura T, Matsui M, Iwata $Y$, et al. A pilot study of tranilast for cardiomyopathy of muscular dystrophy. Intern Med. 2018;57:311-318

30. Sawatani T, Kaneko YK, Doutsu I, et al. TRPV2 channels mediate insulin secretion induced by cell swelling in mouse pancreatic $\beta$ cells. Am J Physiol Cell Physiol. 2019;316:434-443.

31. D'Agnelli S, Arendt-Nielsen L, Gerra MC, et al. Fibromyalgia: genetics and epigenetics insights may provide the basis for the development of diagnostic biomarkers. Mol Pain. 2019;15:1-12.

32. Lévêque $M$, Penna $A$, Le Trionnaire $S$, et al. Phagocytosis depends on TRPV2-mediated calcium influx and requires TRPV2 in lipids rafts: alteration in macrophages from patients with cystic fibrosis. Sci Rep. 2018;8:1-13.

33. Luo J, Hu H. Thermally activated TRPV3 channels. Curr Top Membr 2014;74:325-364.

34. Sherkheli MA, Vogt-Eisele AK, Weber K, et al. Camphor modulates TRPV3 cation channels activity by interacting with critical pore-region cysteine residues. Pak J Pharm Sci. 2013;26:431-438.

35. Doerner JF, Hatt H, Ramsey IS. Voltage- and temperature-dependent activation of TRPV3 channels is potentiated by receptor-mediated PI $(4,5)$ P2 hydrolysis. J Gen Physiol. 2011;137:271-288.

36. Yang P, Zhu MX. TRPV3. Handb Exp Pharmacol. 2014;222:273-291.

37. Cheng $X$, Jin J, Hu L, et al. TRP channel regulates EGFR signaling in hair morphogenesis and skin barrier formation. Cell. 2010;141:331-343.

38. Nilius B, Bíró T. TRPV3: a 'more than skinny' channel. Exp Dermatol. 2013:22:447-452.

39. Lin $Z$, Chen $Q$, Lee $M$, et al. Exome sequencing reveals mutations in TRPV3 as a cause of Olmsted syndrome. Am J Hum Genet 2012;90:558-564.

40. He Y, Zeng $\mathrm{K}$, Zhang $\mathrm{X}$, et al. A gain-of-function mutation in TRPV3 causes focal palmoplantar keratoderma in a Chinese family. J Invest Dermatol. 2015;135:907-909.

41. Broad LM, Mogg AJ, Eberle E, et al. TRPV3 in drug development. Pharmaceuticals (Basel). 2016;9:1-17.

42. Li X, Zhang Q, Fan K, et al. Overexpression of TRPV3 correlates with tumor progression in non-small cell lung cancer. Int J Mol Sci. 2016;17:437-445

43. Kim HO, Cho YS, Park SY, et al. Increased activity of TRPV3 in keratinocytes in hypertrophic burn scars with postburn pruritus. Wound Repair Regen. 2016;24:841-850.

44. Stevens AM, Liu L, Bertovich D, et al. Differential expression of neuroinflammatory mRNAs in the rat sciatic nerve following chronic constriction injury and pain-relieving nanoemulsion NSAID delivery to infiltrating macrophages. Int J Mol Sci. 2019;20:1-24.

45. Ho TC, Horn NA, Huynh T, et al. Evidence TRPV4 contributes to mechanosensitive ion channels in mouse skeletal muscle fibers. Channels (Austin). 2012;6:246-254. 
46. Nilius B, Voets T. The puzzle of TRPV4 channelopathies. EMBO Rep. 2013;14:152-163.

47. TRIP Database-TRPV4; [cited 2020 Sep 9. Available from: http:// trpchannel.org/summaries/TRPV4

48. Cao S, Anishkin A, Zinkevich NS, et al. Transient receptor potential vanilloid 4 (TRPV4) activation by arachidonic acid requires protein kinase A-mediated phosphorylation. J Biol Chem. 2018;293:5307-5322.

49. Garcia-Elias A, Mrkonjić S, Jung C, et al. The TRPV4 channel. Handb Exp Pharmacol. 2014;222:293-319.

- An interesting review on general properties and pharmacological significance of TRPV4 channel.

50. Kitsuki T, Yoshimoto RU, Aijima R, et al. Enhanced junctional epithelial permeability in TRPV4-deficient mice. J Periodontal Res. 2020;55:51-60.

51. Darby WG, Grace MS, Baratchi S, et al. Modulation of TRPV4 by diverse mechanisms. Int J Biochem Cell Biol. 2016;78:217-228.

52. Everaerts W, Nilius B, Owsianik G. The vanilloid transient receptor potential channel TRPV4: from structure to disease. Prog Biophys Mol Biol. 2010;103:2-17.

53. Atobe M. Activation of transient receptor potential vanilloid (TRPV) 4 as a therapeutic strategy in osteoarthritis. Curr Top Med Chem. 2019;19:2254-2267.

54. Olivan-Viguera A, Garcia-Otin AL, Lozano-Gerona J, et al. Pharmacological activation of TRPV4 produces immediate cell damage and induction of apoptosis in human melanoma cells and HaCaT keratinocytes. PLoS One. 2018;13:1-19.

55. Zholos A, Johnson C, Burdyga T, et al. TRPM channels in the vasculature. Adv Exp Med Biol. 2011;704:707-729.

56. Luongo F, Pietropaolo G, Gautier M, et al. TRPM6 is essential for magnesium uptake and epithelial cell function in the colon. Nutrients. 2018;10:1-12.

57. Huang $Y$, Fliegert $R$, Guse $A H$, et al. A structural overview of the ion channels of the TRPM family. Cell Calcium. 2020;85:1-11.

58. Zierler S, Hampe S, Nadolni W. TRPM channels as potential therapeutic targets against pro-inflammatory diseases. Cell Calcium. 2017;67:105-115.

59. Almaraz L, Manenschijn JA, de la Peña E, et al. TRPM8. Handb Exp Pharmacol. 2014;222:547-579.

60. Sousa-Valente J, Andreou AP, Urban L, et al. Transient receptor potential ion channels in primary sensory neurons as targets for novel analgesics. Br J Pharmacol. 2014;171:2508-2527.

61. Ordás $\mathrm{P}$, Hernández-Ortego $\mathrm{P}$, Vara $\mathrm{H}$, et al. Expression of the cold thermoreceptor TRPM8 in rodent brain thermoregulatory circuits. J Comp Neurol. 2019;1:1-23.

62. Liu Y, Mikrani R, He Y, et al. TRPM8 channels: A review of distribution and clinical role. Eur J Pharmacol. 2020;882:173312.

63. Sisco NJ, Helsell CVM, Van Horn WD. Competitive Interactions between PIRT, the cold sensing ion channel TRPM8, and PIP(2) suggest a mechanism for regulation. Sci Rep. 2019;9:1-12.

64. Weyer AD, Lehto SG. Development of TRPM8 antagonists to treat chronic pain and migraine. Pharmaceuticals (Basel). 2017;10:37-46.

65. Hantute-Ghesquier A, Haustrate A, Prevarskaya N, et al. TRPM family channels in cancer. Pharmaceuticals (Basel). 2018;11:1-14.

66. González-Muñiz R, Bonache MA, Martín-Escura C, et al. Recent progress in TRPM8 modulation: an update. Int $\mathrm{J}$ Mol Sci. 2019;20:1-22.

67. Bertamino A, Iraci N, Ostacolo C, et al. Identification of a potent tryptophan-based TRPM8 antagonist with in vivo analgesic activity. J Med Chem. 2018;61:6140-6152.

68. Pérez de Vega MJ, Gómez-Monterrey I, Ferrer-Montiel A, et al. Transient receptor potential melastatin 8 channel (TRPM8) modulation: cool entryway for treating pain and cancer. J Med Chem. 2016;59:10006-10029.

- A comprehensive revision article describing the implication of TRPM8 channels in pain and cancer and compiled the different families of agonists and antagonists.

69. Talavera K, Startek JB, Alvarez-Collazo J, et al. Mammalian transient receptor potential TRPA1 channels: from structure to disease. Physiol Rev. 2020;100:725-803.
70. Chen J, Kang $\mathrm{D}, \mathrm{Xu}$ J, et al. Species differences and molecular determinant of TRPA1 cold sensitivity. Nat Commun. 2013;4:1-7.

71. de Oliveira C, Garami A, Lehto SG, et al. Transient receptor potential channel ankyrin-1 is not a cold sensor for autonomic thermoregulation in rodents. J Neurosci. 2014;34:4445-4452.

72. Knowlton WM, Bifolck-Fisher A, Bautista DM, et al. TRPM8, but not TRPA 1 , is required for neural and behavioral responses to acute noxious cold temperatures and cold-mimetics in vivo. Pain. 2010;150:340-350.

73. Miyake T, Nakamura S, Zhao M, et al. Cold sensitivity of TRPA1 is unveiled by the prolyl hydroxylation blockade-induced sensitization to ROS. Nat Commun. 2016;7:1-10.

74. Koivisto A, Jalava N, Bratty R, et al. TRPA1 antagonists for pain relief. Pharmaceuticals (Basel). 2018;11:1-18.

75. Giorgi S, Nikolaeva-Koleva M, Alarcón-Alarcón D, et al. Is TRPA1 burning down TRPV1 as druggable target for the treatment of chronic pain? Int J Mol Sci. 2019;20:1-20.

76. Kremeyer B, Lopera F, Cox JJ, et al. A gain-of-function mutation in TRPA1 causes familial episodic pain syndrome. Neuron. 2010;66:671-680.

77. Shin SM, Itson-Zoske B, Cai Y, et al. Satellite glial cells in sensory ganglia express functional transient receptor potential ankyrin 1 that is sensitized in neuropathic and inflammatory pain. Mol Pain. 2020;16:1-19.

78. Lu S, Ma S, Wang Y, et al. Mus musculus-microRNA-449a ameliorates neuropathic pain by decreasing the level of KCNMA1 and TRPA1, and increasing the level of TPTE. Mol Med Rep. 2017; 16:353-360.

79. Trevisan G, Materazzi S, Fusi C, et al. Novel therapeutic strategy to prevent chemotherapy-induced persistent sensory neuropathy by TRPA1 blockade. Cancer Res. 2013;73:3120-3131.

80. McGaraughty S, Chu KL, Perner RJ, et al. TRPA1 modulation of spontaneous and mechanically evoked firing of spinal neurons in uninjured, osteoarthritic, and inflamed rats. Mol Pain. 2010;6:1-10.

81. Chen Z, Du S, Kong C, et al. Intrathecal administration of TRPA 1 antagonists attenuate cyclophosphamide-induced cystitis in rats with hyper-reflexia micturition. BMC Urol. 2016;16:33.

82. Mukhopadhyay I, Gomes P, Aranake S, et al. Expression of functional TRPA1 receptor on human lung fibroblast and epithelial cells. J Recept Signal Transduct Res. 2011;31:350-358.

83. Bianchi BR, Zhang XF, Reilly RM, et al. Species comparison and pharmacological characterization of human, monkey, rat, and mouse TRPA1 channels. J Pharmacol Exp Ther. 2012;341:360-368.

84. Heber S, Gold-Binder M, Ciotu Cl, et al. A human TRPA1-specific pain model. J Neurosci. 2019;39:3845-3855.

85. Kort ME, Kym PR. TRPV1 antagonists: clinical setbacks and prospects for future development. Prog Med Chem. 2012;51:57-70.

86. Brederson JD, Kym PR, Szallasi A. Targeting TRP channels for pain relief. Eur J Pharmacol. 2013;716:61-76.

87. Arsenault P, Chiche D, Brown W, et al. NEO6860, modality-selective TRPV1 antagonist: a randomized, controlled, proof-of-concept trial in patients with osteoarthritis knee pain. Pain Rep. 2018;3:1-12.

88. Li S, Bode AM, Zhu F, et al. TRPV1-antagonist AMG9810 promotes mouse skin tumorigenesis through EGFR/Akt signaling. Carcinogenesis. 2011;32:779-785.

89. Park $M$, Naidoo AA, Burns $A$, et al. Do TRPV1 antagonists increase the risk for skin tumourigenesis? A collaborative in vitro and in vivo assessment. Cell Biol Toxicol. 2018;34:143-162.

- Discusses results of a randomized, double-blind, placebocontrolled trial using NEO6860 therapy for osteoarthritis knee pain.

90. Moore C, Gupta R, Jordt SE, et al. Regulation of pain and itch by TRP channels. Neurosci Bull. 2018;34:120-142.

91. Szallasi A, Sheta M. Targeting TRPV1 for pain relief: limits, losers and laurels. Expert Opin Investig Drugs. 2012;21:1351-1369.

92. Iadarola MJ, Gonnella GL. Resiniferatoxin for pain treatment: an interventional approach to personalized pain medicine. Open Pain J. 2013;6:95-107. 
93. Papoiu AD, Yosipovitch G. Topical capsaicin. The fire of a 'hot' medicine is reignited. Expert Opin Pharmacother. 2010;11:1359-1371.

94. Liu Z, Zhu P, Tao Y, et al. Cancer-promoting effect of capsaicin on DMBA/TPA-induced skin tumorigenesis by modulating inflammation, Erk and p38 in mice. Food Chem Toxicol. 2015;81:1-8.

95. Serafini M, Griglio A, Aprile S, et al. Targeting transient receptor potential vanilloid 1 (TRPV1) channel softly: the discovery of passerini adducts as a topical treatment for inflammatory skin disorders. J Med Chem. 2018;61:4436-4455.

96. Devesa Giner I, Genazani APT, Fernandez-Carvajal A, et al. TRPV1 modulator compounds. WO2018206742A1 (2017)

97. Ponsati B, Carreño C, Curto-Reyes V, et al. An inhibitor of neuronal exocytosis (DD04107) displays long-lasting in vivo activity against chronic inflammatory and neuropathic pain. J Pharmacol Exp Ther. 2012;341:634-645.

98. Moreno-Montañés J, Bleau AM, Jimenez Al. Tivanisiran, a novel siRNA for the treatment of dry eye disease. Expert Opin Investig Drugs. 2018;27:421-426.

99. Robbins N, Gilbert M, Kumar M, et al. Probenecid improves cardiac function in patients with heart failure with reduced ejection fraction in vivo and cardiomyocyte calcium sensitivity in vitro. J Am Heart Assoc. 2018;7:1-11.

100. Darakhshan S, Pour AB. Tranilast: a review of its therapeutic applications. Pharmacol Res. 2015;91:15-28.

101. Iwata Y, Matsumura T. Blockade of TRPV2 is a novel therapy for cardiomyopathy in muscular dystrophy. Int J Mol Sci. 2019;20:3844-3857.

102. Siveen KS, Prabhu KS, Parray AS, et al. Evaluation of cationic channel TRPV2 as a novel biomarker and therapeutic target in leukemia-implications concerning the resolution of pulmonary inflammation. Sci Rep. 2019;9(1):1554-1562. .

103. Biosciences H Compounds for modulating TRPV3 function. WO2007056124. 2007.
104. Glenmark Pharmaceuticals SA Chromane derivatives as TRPV3 modulators. WO2010004379. 2010.

105. Al-Harrasi A, Csuk R, Khan A, et al. Distribution of the anti-inflammatory and anti-depressant compounds: incensole and incensole acetate in genus Boswellia. Phytochemistry. 2019;161:28-40.

106. Stewart JM TRPV3 Agonists for the Treatment of Skin Conditions US20150250699 (2015)

107. Lawhorn BG, Brnardic EJ, Behm DJ. Recent advances in TRPV4 agonists and antagonists. Bioorg Med Chem Lett. 2020;30:1-6.

108. Andersen HH, Gazerani P, Arendt-Nielsen L. High-concentration L-menthol exhibits counter-irritancy to neurogenic inflammation, thermal and mechanical hyperalgesia caused by trans-cinnamaldehyde. J Pain. 2016;17(8):919-929.

109. Tolcher A, Patnaik A, Papadopoulos K, et al. 376 Preliminary results from a Phase 1 study of D-3263 $\mathrm{HCl}$, a TRPM8 calcium channel agonist, in patients with advanced cancer. EJC Suppl. 2010;8(7):119.

110. Alcalde I, Îñigo-Portugués A, González-González O, et al. Morphological and functional changes in TRPM8-expressing corneal cold thermoreceptor neurons during aging and their impact on tearing in mice. J Comp Neurol. 2018;526:1859-1874.

111. Andrews MD, Af Forselles K, Beaumont K, et al. Discovery of a selective TRPM8 antagonist with clinical efficacy in cold-related pain. ACS Med Chem Lett. 2015;6:419-424.

112. Horne DB, Biswas K, Brown J, et al. Discovery of TRPM8 antagonist (S)-6-(((3-Fluoro-4-(trifluoromethoxy)phenyl)(3-fluoropyridin-2-yl) methyl)carbamoyl)nicotinic Acid (AMG 333), a clinical candidate for the treatment of migraine. J Med Chem. 2018;61 (18):8186-8201.

113. Miller $F$, Björnsson $M$, Svensson $O$, et al. Experiences with an adaptive design for a dose-finding study in patients with osteoarthritis. Contemp Clin Trials. 2014;37(2):189-199. 